\title{
Percepción de los estudiantes de ciencias de la salud acerca de su formación en promoción de la salud
}

\author{
Perception of health sciences students about their training in health promotion
}

\author{
Gladys Santos-Falcón ${ }^{1}$, María Infante-Contreras ${ }^{1}$, María Arcaya-Moncada ${ }^{1}$, \\ Carlos Márquez-Cabezas², Maribel Gil1, Yelin Ramírez ${ }^{3}$ \\ ${ }^{1}$ Departamento Académico de Enfermería, Facultad de Medicina, Universidad Nacional Mayor de San Marcos. Lima, Perú. \\ ${ }^{2}$ Departamento Académico de Medicina Preventiva, Facultad de Medicina, Universidad Nacional Mayor de San Marcos. Lima, Perú. \\ ${ }^{3}$ Interna de la Escuela Académico Profesional de Enfermería, Facultad de Medicina, Universidad Nacional Mayor de San Marcos. Lima, Perú.
}

\begin{abstract}
Resumen
Objetivos: Identificar la percepción sobre su formación en promoción de la salud de estudiantes de una Facultad de Medicina Peruana. Diseño: Estudio tipo cuanticualitativo. Institución: Facultad de Medicina, Universidad Nacional Mayor de San Marcos, Lima, Perú. Participantes: Estudiantes del penúltimo año de estudios de cinco escuelas académico profesionales (EAPs) de la Facultad de Medicina, UNMSM. Intervenciones: Se utilizó dos técnicas de recolección de datos: una entrevista estructurada, con un cuestionario tipo Lickert modificado, y grupos focales. La población estuvo conformada por los estudiantes del penúltimo año de estudios de las cinco EAPs de la Facultad de Medicina (489 estudiantes), y la muestra la conformaron 127 estudiantes. Principales medidas de resultados: Cuantificar la percepción de los estudiantes de las cinco escuelas académico profesionales sobre su formación, en relación a las estrategias de la promoción de la salud propuestas por la Carta de Ottawa. Resultados: La percepción de los estudiantes sobre su formación en relación a la promoción de la salud ha sido medianamente favorable, con tendencia a favorable en dos escuelas: Escuela de Enfermería en las estrategias de creación de ambientes favorables para la salud y reforzamiento de la acción comunitaria, con $70,5 \%$ y $58,3 \%$, respectivamente. Asimismo, los estudiantes de la Escuela de Nutrición tuvieron una percepción favorable en la dimensión de implementación de políticas públicas, en $60,9 \%$. Cabe resaltar que, solo en la dimensión de - habilidades personales - la percepción de los estudiantes fue desfavorable en un porcentaje considerable en dos escuelas: Escuela de Medicina, en 23,4\%, y Tecnología Médica, en $20,4 \%$ de los estudiantes. Con los datos cualitativos se estableció siete categorías de análisis: 1) valorando la promoción de la salud; 2) relacionando la promoción de la salud con la atención comunitaria; 3) sintiéndose motivados para promocionar la salud; 4) valorando el reforzamiento comunitario; 5) considerando que la Facultad de Medicina debe promover la salud; 6) considerando los factores que dificultan la promoción de la salud; y 7) valorando la interdisciplinaridad para la promoción de la salud. Conclusiones: Los estudiantes de la Facultad de Medicina tienen una percepción diversificada en relación a su formación en promoción de la salud, según las estrategias propuestas por la Carta de Ottawa, por lo que se hace necesario mejorar esta formación en las cinco escuelas con el objetivo de unificar la formación de estos profesionales para que desarrollen la promoción de la salud interdisciplinariamente y así poder enfrentar los complejos problemas de salud presentes en nuestra sociedad.

Palabras clave: Promoción de la salud; estudiantes del área de la salud; facultades de medicina.
\end{abstract}

\begin{abstract}
Objectives: To identify a Peruvian Faculty of Medicine students' perception on training in health promotion. Design: Quantitative-qualitative study. Institution: Faculty of Medicine, Universidad Nacional Mayor de San Marcos, Lima, Peru. Participants: Faculty of Medicine's five academic professional schools penultimate year students. Interventions: Two data collection techniques -an interview with a modified Lickert questionnaire and focus group- were applied to 127 students. Main outcome measures: Quantification of the students' perception on their training on health promotion strategies in relation to Ottawa's Letter proposition on health promotion. Results: The students' perception on their health promotion strategies training was moderately favorable, with tendency to be quite favorable in two them: School of Nursing in creating supportive environments for health and strengthening community action, with $70,5 \%$ and $59,3 \%$ respectively; School of Nutrition students had a completely favorable perception on implementing public policies, in $60,9 \%$. It should be noted that only in one dimension (personal abilities) the students' perceptions was unfavorable in two schools: Medical School, in 23,4\%, and Medical Technology, in 20,4\%. With the qualitative data we structured seven analysis categories: 1) appreciating health promotion; 2) linking health promotion with community care; 3 ) being motivated to promote health; 4) appreciating community strengthening; 5) determining the Faculty should promote health; 6) considering factors that hinder health promotion; and, 7) appreciating interdisciplinary involvement in health promotion. Conclusions: The Faculty of Medicine students had varied perception in relation to health promotion formation, according to strategies proposed by Ottawa's Letter. It will be necessary to unify and improve training in order to develop interdisciplinary health and confront health complex problems present in our society.
\end{abstract}

Key words: Health promotion; students, health occupations; schools, medical.

\section{INTRODUCCIÓN}

En los países del tercer mundo, entre ellos el Perú, la realidad en salud es compleja, pues las inequidades típicas del modelo de desarrollo social mantienen presentes un perfil epidemiológico de enfermedades asociadas a la pobreza (como enfermedades infecto-contagiosas, desnutrición, tuberculosis, entre otras), presentándose también enfermedades propias de países desarrollados (enfermedades crónicodegenerativas, coronarias, obesidad, entre otras) Frente a esta realidad, el discurso y la práctica sanitaria hegemónica, basados en el modelo biomédico, entran en crisis, mostrándose ineficaces para el enfrentamiento de la complejidad de los cuadros sanitarios presentes en el siglo XXI ${ }^{(1)}$.
El presente estudio surge de las reflexiones realizadas sobre la temática por docentes de enfermería comprometidos con la formación de profesionales que respondan a las necesidades de salud de la población. Creemos que la realidad en salud requiere que sus profesionales sean formados con conciencia de los cambios y del contexto en general. En ese sentido, concordamos con Morin 
cuando dice que: "existe inadecuación cada vez más amplia, profunda y grave, entre, de un lado, los saberes desunidos, divididos, compartimentalizados y, del otro, las realidades o problemas cada vez más multidisciplinares, transversales, multidimensionales, y planetarios" ${ }^{(2,3)}$.

Según la Organización Mundial de la Salud, las condiciones y los recursos fundamentales para la salud son paz, vivienda, educación, alimentación, renta, eco-sistema estable, recursos sustentables, justicia social y equidad, afirmando que el incremento en las condiciones de salud requiere una base sólida en estos prerequisitos básicos. Está asociada, también, a un conjunto de valores, como solidaridad, democracia, ciudadanía, desarrollo, participación conjunta, entre otros ${ }^{(4)}$.

El concepto moderno de promoción de la salud, así como su práctica, surge y se desarrolla de forma más vigorosa en los últimos 20 años en los países desarrollados, particularmente en el Canadá, en los Estados Unidos y en los países de Europa Occidental. Tres importantes conferencias internacionales sobre el tema, realizadas entre 1986 y 1991, en Ottawa (1986), Adelaide (1988) y Sundsval (1991), establecieron las bases conceptuales y políticas contemporáneas de la promoción de la salud. Las siguientes conferencias se realizaron en Jakarta, en 1997, en México, en el 2000, y en Bangkok - Tailandia, en el $2005^{(5)}$.

La Carta de Ottawa define la promoción de la salud como: "El proceso de capacitación de la comunidad para actuar en la mejoría de su calidad de vida y salud, incluyendo una mayor participación en el control de este proceso" ${ }^{(6)}$. Partiendo de una concepción amplia del proceso salud-enfermedad y de sus determinantes, la promoción de la salud propone la articulación de saberes técnicos y populares, así como la movilización de recursos institucionales y comunitarios, públicos y privados para su enfrentamiento y resolución ${ }^{(7)}$.

La promoción de la salud, entendida de una forma amplia y no solamente como un momento anterior a la enfermedad, debe ser vista considerando la autonomía de las personas, en su contexto político y cultural, como sujetos capaces de mejorar, generando transformaciones en sus condiciones de vida ${ }^{(8)}$. Esto implica que los futuros profesionales de la salud deberían estar preparados para asumir un papel más activo y dinamizador en el fortalecimiento de la prevención de la enfermedad y promoción de la salud, y poder construir un nuevo modelo de atención en salud, que no solo se oriente a la educación sanitaria, sino que tenga una orientación más amplia, considerando los determinantes de la salud; responderían así, a las necesidades de salud de la población peruana.

Se observa que, a pesar de considerarse una prioridad la promoción de la salud, no hay inversión significativa en capacitación de los recursos humanos, implementación de los servicios del primer nivel de atención e incluso en el reconocimiento en el sistema de salud de las actividades específicas de la labor preventivo-promocional que se desarrolla en los servicios de salud del primer nivel de atención.

Asimismo, la formación universitaria se basa esencialmente en lo recuperativo, privilegiando la actividad asistencial hospitalaria, por lo que es necesario reorientar el proceso formativo en salud hacia lo preventivo promocional, en razón a que de esta manera se estaría respondiendo a la problemática sanitaria y así contribuir de alguna manera al mejoramiento de la calidad de vida de las poblaciones del país.

Los objetivos generales que se plantearon para el presente estudio fueron identificar la percepción del estudiante del penúltimo año de estudios de la Facultad de Medicina sobre su formación en promoción de la salud e identificar sus perspectivas de desarrollo profesional en esta área. Los objetivos específicos consistieron en identificar la percepción sobre la formación de los estudiantes, según las cinco directrices que considera la Carta de Otawa, para el desarrollo de la promoción de la salud: a) implementación de politicas públicas saludables; b) refuerzo de la acción comunitaria; c) desarrollo de habilidades personales; d) creación de ambientes saludables; y, e) reorientación de los servicios de salud para la promoción de la salud. Además, se tuvo el objetivo específico de describir las perspectivas de desarrollo profesional en el área de promoción de la salud del estudiante de la Facultad de Medicina.

\section{MÉTODOS}

Se utilizó un tipo de estudio descriptivo, con abordaje cuanti-cualitativo. La población estuvo constituida por los estudiantes del penúltimo año de estudios de las cinco escuelas profesionales de una Facultad de Medicina, con un total de 489 estudiantes. La muestra estuvo compuesta por 127 estudiantes, distribuidos de la siguiente manera: 37 de la Escuela de Medicina Humana, 21 de la Escuela de Obstetricia, 22 de la Escuela de Enfermería, 31 de la Escuela de Tecnología Médica y 16 de la Escuela de Nutrición. El periodo de recolección de datos fue de julio a octubre del 2008, en dos momentos; en el primer momento, se recolectó los datos cuantitativos relacionados con la percepción de los estudiantes, para lo cual se aplicó un cuestionario con escala tipo Likert, conformada por 33 ítems; los resultados de este instrumento fueron calificados como percepción favorable, medianamente favorable y percepción desfavorable. En el segundo momento, se recolectó los datos cualitativos mediante grupos focales, para estudiar los aspectos subjetivos en relación a las perspectivas del estudiante sobre su desarrollo profesional en el área de promoción de la salud.

Estos datos cualitativos fueron transcritos, agrupándolos luego en unidades de significado, asignándose un código a cada unidad y agrupándose los códigos similares para constituir las categorías del estudio que fueron posteriormente analizadas e interpretadas.

\section{RESULTADOS}

Las características de los participantes en la investigación en cuanto a edad y sexo fueron las siguientes: 72 menores de 25 años, 51 entre 25 y 30 años y 4 mayores de 30 años; 76 fueron del sexo femenino y 51 del masculino.

En la figura 1 sobre la dimensión políticas públicas, se observa que los estudiantes de las escuelas de Nutrición 
y Enfermería tuvieron una percepción favorable en $60,9 \%$ y $55,7 \%$, respectivamente. En tanto que se encontró una percepción medianamente favorable en las Escuelas de Obstetricia, con 64\%, seguida por la Escuela de Medicina, con 54,1\%; finalmente, la Escuela de Tecnología Médica, con 53,8\%.

La incorporación en la formación de los estudiantes de ciencias de la salud de los aspectos de políticas públicas saludables constituye una necesidad, ya que los estudiantes deben estar conscientes que la promoción de la salud exige la acción coordinada de todos los implicados: los gobiernos, los sectores sanitarios y otros sectores sociales y económicos, las organizaciones benéficas, las autoridades locales, la industria y los medios de comunicación, y deben estar preparados para actuar como intermediarios entre todos estos organismos para la implementación de planes y programas necesarios a una mejor atención de salud de la población.

En la figura 2, referida a la dimensión de reforzamiento de la acción comunitaria, se puede observar que existieron diferencias marcadas entre escuelas sobre esta estrategia; así, los estudiantes que tuvieron una percepción favorable pertenecían a las Escuelas de Enfermería, con 58,3\%, y Nutrición, con 48,9\%. Y con percepción medianamente favorable se encontraban la Escuela de Obstetricia, con 65,9\%, y la Escuela de Medicina, con 57,7\%; en tanto que la Escuela de Tecnología Médica tuvo el mayor porcentaje de percepción desfavorable, con 20,9\%, seguido de la Escuela de Medicina, con 17,6\%.

Estos resultados nos muestran que no existe uniformidad en la formación de los profesionales de salud sobre aspectos tan importantes como es el reforzamiento de la acción comunitaria, la que debe ser una prioridad, dada la realidad del país, que exige un empoderamiento de los miembros de la comunidad en el cuidado de su salud y así poder mejorar su calidad de vida.

En la figura 3, sobre la percepción en relación al desarrollo de habilidades personales para la promoción de la salud, se observa una percepción favorable en $46,9 \%$ en la Escuela de Enfermería y $43,8 \%$ en la de Nutrición; en tanto que fue desfavorable en $23,4 \%$ en la Escuela

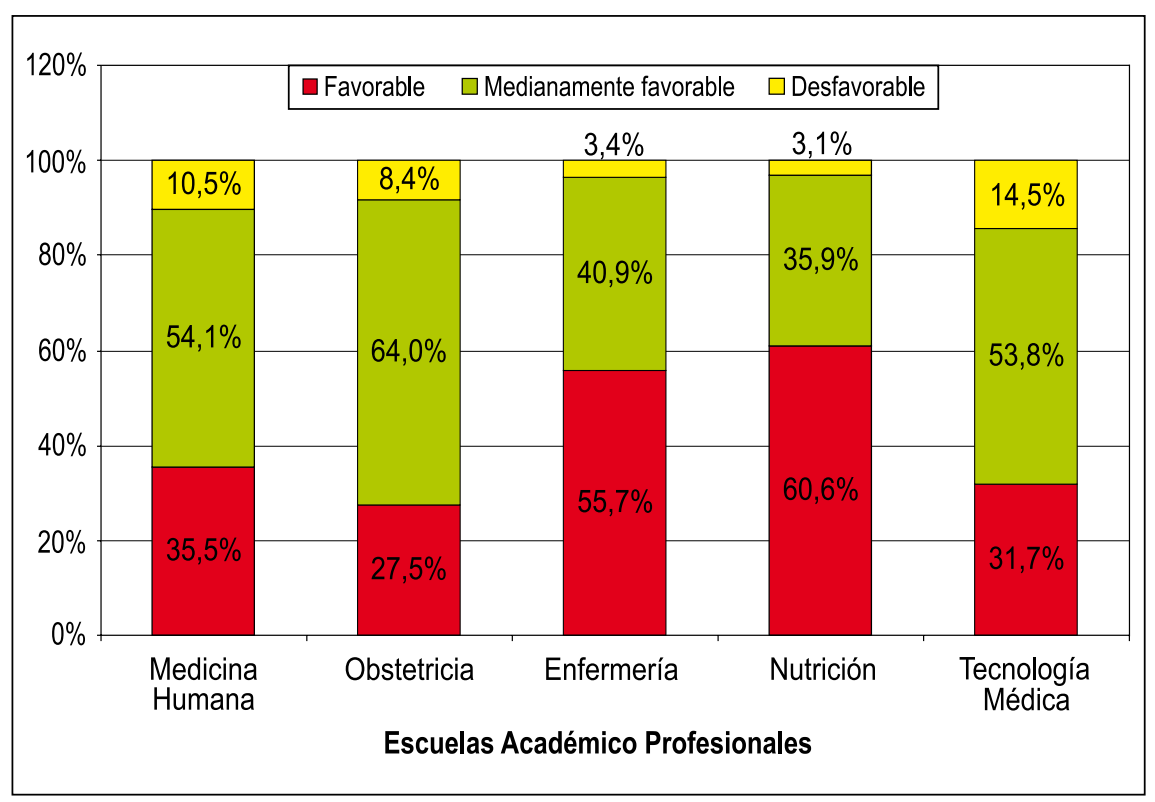

Figura 1. Percepción de los estudiantes de las Escuelas Académico Profesionales sobre su formación en promoción de la salud, en la dimensión de políticas públicas. de Medicina y 20,4\% en la Escuela de Tecnología Médica; y fue medianamente favorable en la Escuela de Obstetricia, con $58,7 \%$.

Es necesario que los futuros profesionales de salud internalicen que la población debe desarrollar habilidades personales y redes sociales que le permitan generar cambios y tener un mayor control sobre los determinantes que influyen en su salud; por lo que es necesario dar a las personas las oportunidades, conocimientos, y acceso a los servicios y recursos para generar en ellos su capacidad de autocuidado de su salud y la de su familia. En el estudio se encontró que esta dimensión es la más débil en relación a la percepción de los estudiantes. Ante tal escenario, la entidad formadora debe plantearse el reto de formar profesionales de la salud

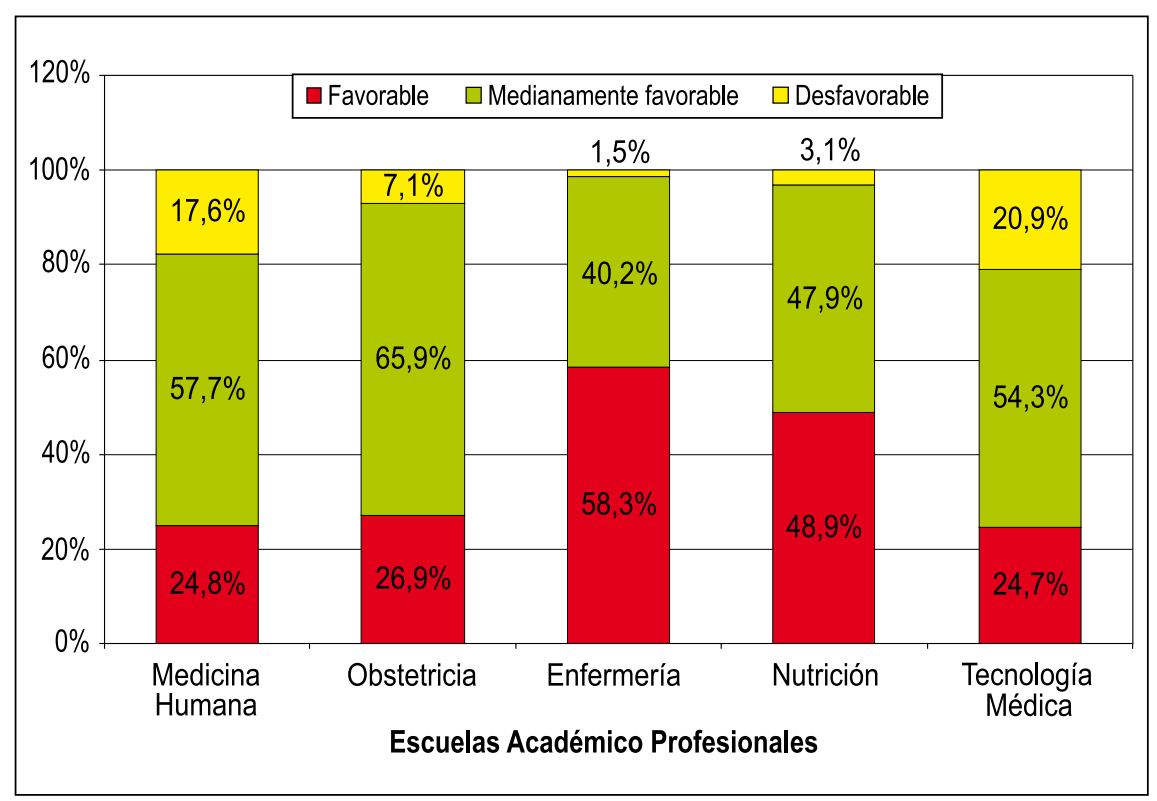

Figura 2. Percepción de los estudiantes de las Escuelas Académico Profesionales sobre su formación en promoción de la salud, en la dimensión de reforzar la acción comunitaria. 


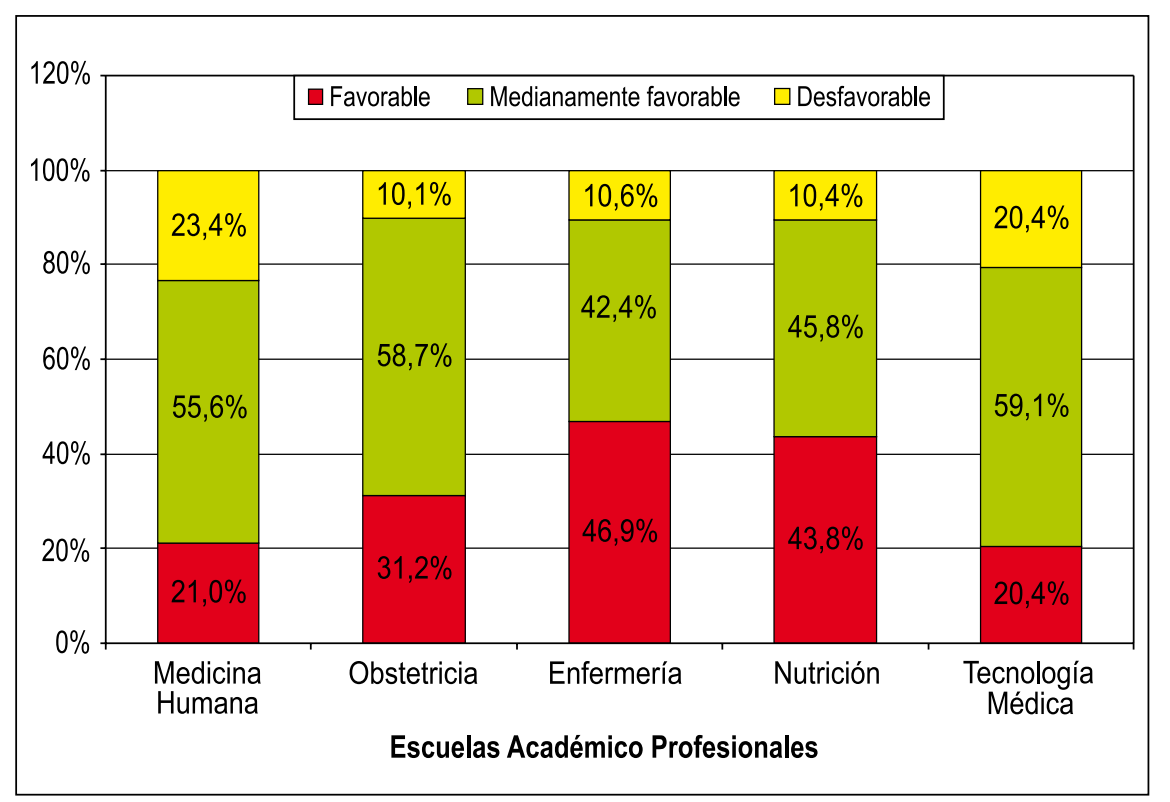

Figura 3. Percepción de los estudiantes de las Escuelas Académico Profesionales sobre su formación en promoción de la salud, en la dimensión de habilidades profesionales.

competentes en esta estrategia de la promoción de la salud.

En la figura 4, referida a la percepción de las estudiantes sobre la dimensión de ambientes favorables, se observa que el mayor porcentaje de percepción favorable se encontró en las escuelas de Enfermería, con 70,5\%, seguida de la Escuela de Nutrición, con $62,5 \%$, teniendo ambas escuelas $0 \%$ de percepción desfavorable;

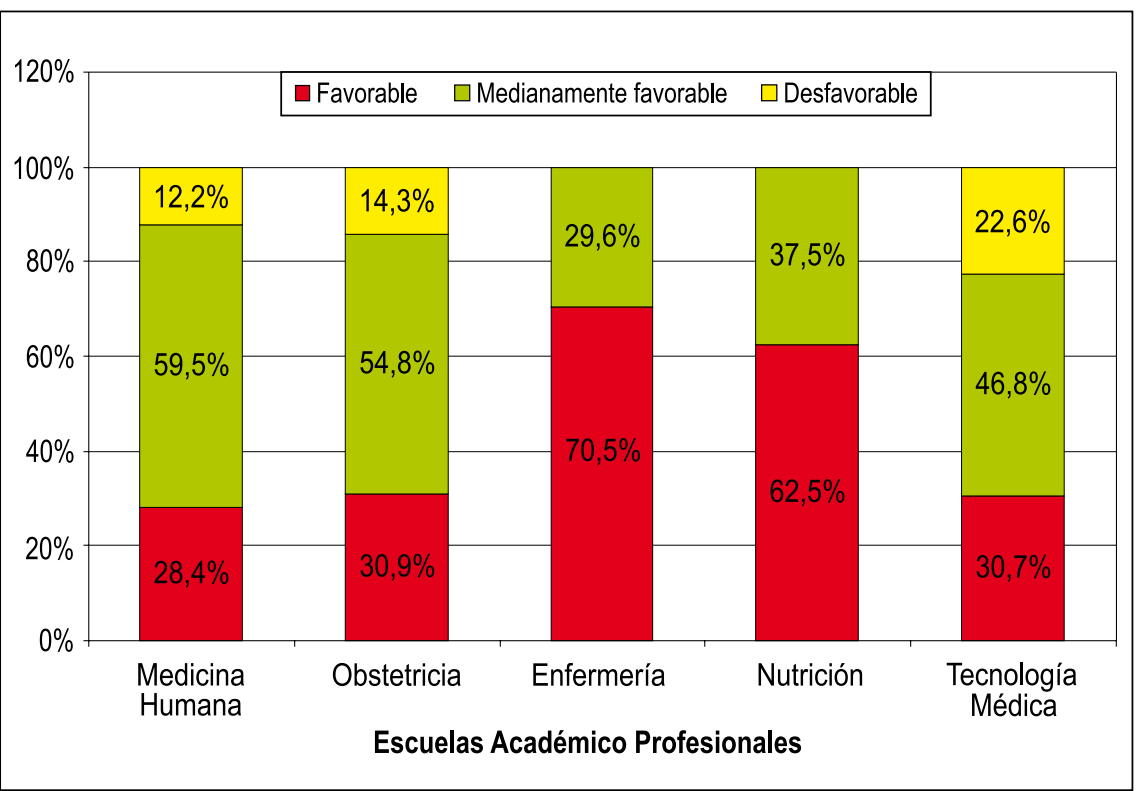

Figura 4. Percepción de los estudiantes de las Escuelas Académico Profesionales sobre su formación en promoción de la salud, en la dimensión de ambientes favorables. sirve de base para un abordaje socioecológico de la salud. Sin embargo, encontramos que solo las escuelas de Enfermería y Nutrición alcanzaron resultados favorables en esta dimensión, por lo que es necesario uniformizar las estrategias curriculares para el desarrollo de esta dimensión en todas las escuelas de la Facultad, considerando los nuevos escenarios para la práctica de la promoción de la Salud, como hogares, comunidades, centros educativos y laborales, con fines de ofrecer nuevas experiencias de aprendizaje sobre promoción de ambientes saludables.

En la figura 5, relacionada a la percepción sobre la reorientación de los servicios de salud, se observa diferencias significativas en las diferentes escuelas de la Facultad de Medicina. Así, la Escuela de Nutrición alcanzó una percepción favorable de 51,9\% seguida de la Escuela de Enfermería, con 43,2\%, encontrándose la percepción más baja -considerada como desfavorable- en las escuelas de Tecnología Médica y Medicina, en las cuales se obtuvo los porcentajes de 25,8\% y 23,9\%, respectivamente.

La reorientación de los servicios de salud se refiere a que la formación de los futuros profesionales de la salud debería incidir en la incorporación de la promoción de la salud, desarrollando competencias que fortalezcan el trabajo multidisciplinario, empoderamiento de los actores sociales del nivel local, revalorización del conocimiento popular y sus prácticas de cuidado, con una perspectiva intercultural.

En el análisis cualitativo de los datos, se obtuvo siete categorías, que fueron: 1 ) valorando la promoción de la salud; 2) relacionando la promoción de la salud con la atención comunitaria; 3) sintiéndose motivados para promocionar la salud; 4) valorando el reforzamiento comunitario; 5) considerando que la Facultad de Medicina debe promover la salud; 6) considerando los factores que dificultan la formación en promoción de la salud; $y$, 7) valorando la interdisciplinaridad para desarrollar la promoción de la salud. Ellas fueron interpretadas a la luz de los autores de la corriente de la Nueva Promoción de la Salud. 


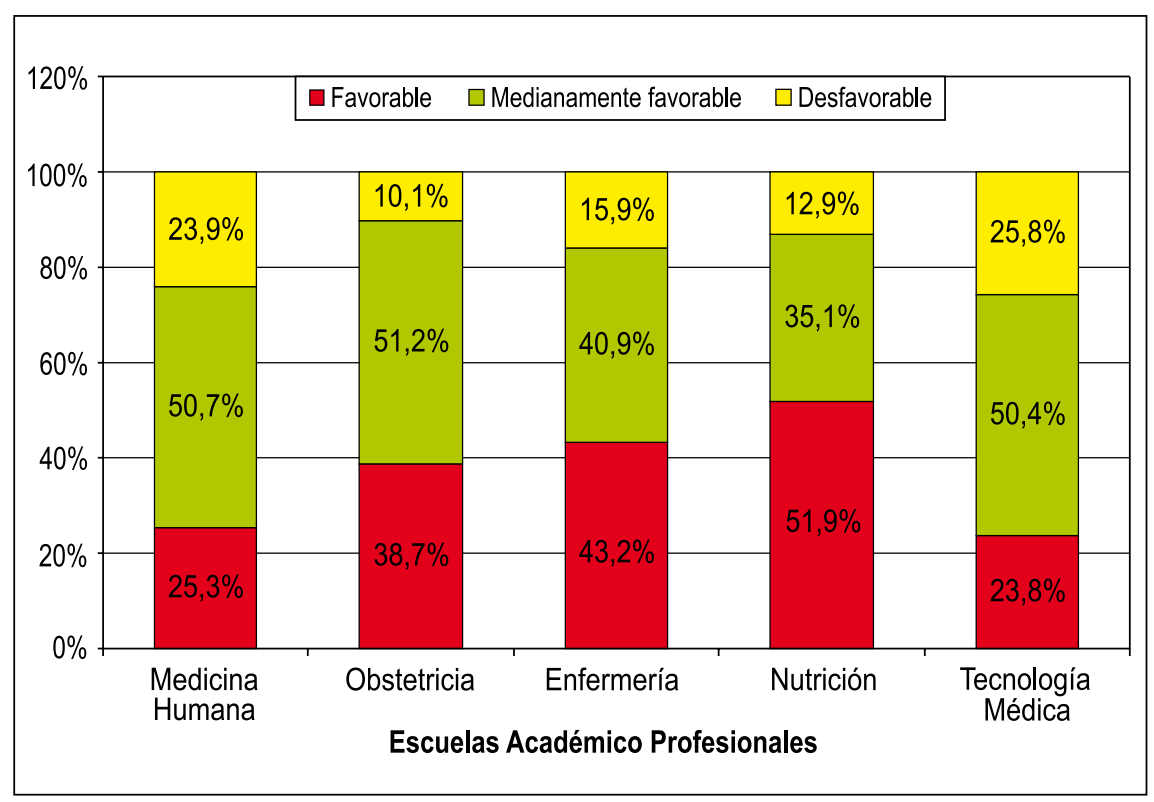

Figura 5. Percepción de los estudiantes de las Escuelas Académico Profesionales sobre su formación en promoción de la salud, en la dimensión de reorientación de los servicios de salud.

\section{DISCUSIÓN}

Encontramos que la percepción del estudiante del pre-internado de la Facultad de Medicina, sobre su formación en este tema, en las diversas escuelas, va de lo medianamente favorable a lo favorable, en la mayoría de ellas, existiendo algunas fortalezas, como por ejemplo, el hecho que se considere a las poblaciones vulnerables -niños, ancianos y discapacitadoscomo sujetos prioritarios en la adopción de políticas de promoción de la salud, y que se identifique a los factores condicionantes de la salud en la formación de los profesionales en esta área.

La percepción depende de la ordenación, clasificación y elaboración de sistemas de categorías con los que se compara los estímulos que el sujeto recibe a través de sus experiencias sensoriales. La manera de clasificar lo percibido es moldeada por circunstancias sociales, como la cultura, el grupo, la clase social a la que se pertenece, y van a influir sobre las formas como es concebida la realidad, las cuales son aprendidas y reproducidas por los sujetos sociales ${ }^{(9)}$.

Varios autores identifican la promoción de la salud como una acción global que compete a individuos, comunidades y otros sectores públicos y no solo como una responsabilidad del sector salud ${ }^{(10,11)}$.
En ese sentido, se manifiesta como una fortaleza en la formación, especialmente en las escuelas de Enfermería y Nutrición, seguida de la Escuela de Medicina, evidenciándose en la percepción del estudiante la importancia de establecer redes de apoyo con la clase dirigente y otros sectores de la comunidad y no concebirla solo como responsabilidad del sector salud.

Los espacios formativos, especialmente los universitarios, deberán preparar a los futuros profesionales para actuar como mediadores entre la población y los niveles directivos, a manera de integrar los esfuerzos, con un enfoque de intersectorialidad como una nueva forma de trabajar, de gobernar y de desarrollar políticas públicas, orientadas a la promoción de la salud, para lograr la mejoría de la calidad de vida de la población.

En el estudio encontramos que el estudiante del pre-internado de la Facultad de Medicina consideró que su formación en promoción de la salud ha sido medianamente favorable en la mayoría de las dimensiones y escuelas, con tendencia a ser totalmente favorable, como es el caso de enfermería, en las dimensiones de reforzamiento de la acción comunitaria y creación de ambientes favorables para la salud. Asimismo, los estudiantes de la Escuela de Nutrición tuvieron una percepción favorable en las dimensiones de implementación de políticas públicas y reorientación de los servicios de salud. Estos resultados evidencian que existen diferencias de enfoque en la formación sobre promoción de la salud, siendo necesario su uniformización y trabajo interdisciplinario, inclusive a lo largo del proceso formativo.

Otro de los aspectos identificados fue que la dimensión más débil fue la de reforzamiento de habilidades personales de los miembros de la comunidad, la cual requeriría ser trabajada en todas las escuelas, ya que se obtuvo porcentajes desfavorables en $23,4 \%$, en la Escuela de Medicina, y 20,4\% en la Escuela de Tecnología Médica. Solo las escuelas de Enfermería y Nutrición alcanzaron una percepción favorable de $46,9 \%$ y $43,8 \%$, respectivamente.

El tema de la interdisciplinaridad afloró en el proceso del estudio durante el desarrollo de los grupos focales, siendo considerado este aspecto como muy importante para el desarrollo de la promoción de la salud, resultados que se asemejan a los obtenidos por Santos, en un estudio realizado con estudiantes de Enfermería en una universidad brasileña ${ }^{(12)}$, en el cual se evidenció la dificultad existente para la formación interdisciplinar de los profesionales de salud. Sin embargo, existe la concientización de los estudiantes y los docentes en cuanto a la importancia de aprender a trabajar en forma conjunta.

La realidad sanitaria exige que en los centros universitarios se formen profesionales de salud conscientes de su complejidad y de la necesidad del trabajo interdisciplinario, para actuar en el mejoramiento de las condiciones de vida de la población, aportando desde su saber los conocimientos que lleven a alcanzar mejores niveles de vida de la población, tal como lo señalan la Organización Mundial de la Salud, la Declaración de los Derechos Humanos, entre otros.

Otro fundamento importante para una práctica interdisciplinar entre los profesionales de la salud consiste en la tentativa de incentivar el diálogo con otras formas de conocimiento, y esa tentativa posibilitará la interpretación y la articulación entre los diferentes saberes ${ }^{(13)}$. 
Consideramos que, siendo la Facultad de Medicina una institución formadora de cinco carreras profesionales de salud, debería propiciar la interdisciplinaridad desde la formación, debiéndose planificar programas de promoción de la salud conjuntamente con los estudiantes de las cinco escuelas que la conforman, incluyendo también a los docentes. De esta manera, los estudiantes aprenderían a trabajar en equipo y lo continuarían durante su vida profesional, dando así, una atención integral a los usuarios.

Es preciso tener en cuenta que la percepción emana desde la conciencia del sujeto; de allí los resultados observados, los cuales son diferentes en las diversas escuelas y aún en los sujetos de una misma escuela. Esto responde a una de las características de la percepción, que es un proceso particular, es decir que cada grupo social tiene su propio mundo, o sus propios mundos en momentos diferentes.

La manera como la persona clasifica lo que percibe es moldeada por diversas circunstancias y que van a influir sobre las formas como es concebida la realidad, las cuales son aprendidas y reproducidas por los sujetos sociales. Este proceso de moldeado se hace a través del aprendizaje mediante la socialización del individuo en el grupo del que forma parte, de manera implícita y simbólica. Por eso, se considera que uno de los elementos importantes que definen a la percepción es el reconocimiento de las experiencias cotidianas, lo que permite evocar experiencias y conocimientos previamente adquiridos a lo largo de la vida, con los cuales se compara las nuevas experiencias, lo que permite identificarlas $\mathrm{y}$ aprehenderlas para interactuar en el entorno ${ }^{(14)}$.

De allí los datos cualitativos obtenidos en el estudio, siendo una de las sugerencias de los estudiantes que, para una adecuada formación, se debe fomentar los aspectos relacionados con la promoción de la salud, teniendo en cuenta que constituye una necesidad del país, resultando más económico direccionar la atención de salud hacia los aspectos preventivopromocionales y con ello contribuir al mejoramiento de la calidad de vida de los peruanos.
Estos datos contrastan con los obtenidos en el estudio realizado por Ramírez para establecer la percepción de los internos de medicina sobre la atención primaria, quien encontró que la gran mayoría de los internos de medicina deseaba desempeñar su labor asistencial en el tercer o cuarto nivel de atención y solo un reducido $11,1 \%$ mostró alguna inclinación por el primer nivel de atención ${ }^{(15)}$.

En ese sentido, es necesario replantear la formación de los profesionales de la salud de nuestro país, tal como Paim nos dice: "la (re)conceptualización de las necesidades sociales de salud apunta para la pertinencia de estructurar conocimientos y habilidades que conformen competencias profesionales orgánicamente vinculadas a las exigencias de nuestros tiempos" (16).

Concluimos que la percepción del estudiante del penúltimo año (preinternado) de la Facultad de Medicina emana de su proceso de aprendizaje en promoción de la salud a lo largo de su formación. Cabe resaltar que este tema es un pilar fundamental en la atención de salud para el logro del mejoramiento de la calidad de vida y el desarrollo humano de las poblaciones, y por lo tanto deberá estar presente en los planes curriculares de la Facultad.

La formación dirigida a la promoción de la salud no es un proceso uniforme en las escuelas de la Facultad de Medicina y posiblemente se deba a la escasa integración de estos aspectos en los planes curriculares y a las estrategias de enseñanza que son utilizadas en el proceso formativo.

Se hace necesario desarrollar propuestas operativas con un enfoque interdisciplinario, procurando una mayor participación comunitaria, en la cual actúen los estudiantes, formando así profesionales conocedores de la realidad sanitaria de nuestro país y comprometidos con su formación como personas y ciudadanos.

\section{REFERENCIAS BIBLIOGRÁFICAS}

1. Lefevre F, Lefevre AC. Promoção de saúde: a negação da negação. Rio de Janeiro: Vieira \& Lent; 2004.
2. Morin E. A cabeça bem feita. Repensar a Reforma, Reformar o Pensamento. $3^{\mathrm{a}}$ ed. Rio de Janeiro: Bertrand-Brasil; 2001.

3. Morin E. Ciência com consciência. $7^{a}$ ed. Rio de Janeiro: Bertrand-Brasil; 2003.

4. World Health Organization - WHO. The Ottawa Charter for Health Promotion. Ottawa, Canada, November, 1986.

5. Buss PM. Uma Introdução ao Conceito de Promoção da Saúde. En: Czeresnia D, Machado C (Org). Promoção da saúde: conceitos, reflexões, tendências. Rio de Janeiro: Fiocruz; 2003.

6. Brasil, Ministério da Saúde/Secretaria de Políticas de Saúde. Projeto Promoção da Saúde. As Cartas de Promoção da Saúde. Brasília, DF, 2002.

7. Mendes EV. Uma agenda para a Saúde. São Paulo: Hucitec; 1996.

8. Verdi M, Caponi S. Reflexões sobre a promoção da saúde numa perspectiva bioética. Texto Contexto Enferm. 2005;14(1):82-8.

9. Xirau J. Obras Completas I. Escritos Fundamentales. Madrid: Antrophos Editorial: 1998.

10. Carvalho SR. Saúde coletiva e promoção da saúde. Sujeito e mudança. São Paulo: Hucitec; 2005.

11. Heidemann ISB. A promoção da saúde e a concepção dialógica de Freire: possibilidades de sua inserção e limites no processo de trabalho das equipes de Saúde da Família, 2006. 296p. Tesis (Doctorado en Enfermería) - Programa de Pos-Graduación en Enfermería, Universidade de São Paulo, SP, Riberão Preto, 2005.

12. Santos FG. Promovendo a saúde: desvelando ordem, desordem e auto-organização de um novo modo de ensinar e aprender para cuidar em saúde. Tesis (Doctorado en Enfermería) - Programa de Pos-Graduación en Enfermería, Universidade Federal de Santa Catarina, Florianópolis, Brasil, 2007.

13. Berardinelli L, Santos M. Repensando a interdisciplinaridade e o ensino de enfermagem. Texto Contexto Enferm. 2005;14(3).

14. López-lbor JJ, Ortiz A, López-lbor MA. Lecciones de Psicología Médica. Madrid: Masson SA; 1999.

15. Ramírez M. Expectativas profesionales de internos de medicina y su inclinación por la atención primaria de salud. Trabajo presentado como Tesis para optar al Título Profesional de Médico Cirujano en la Facultad de Medicina -UNMSM. Lima, Perú, 2006.

16. Paim JS, Almeida FN. A crise da saúde pública e a utopia da saúde coletiva. Salvador, BA, Brasil: Casa da Qualidade Editora; 2000.

Manuscrito recibido el 19 de octubre de 2009 y aceptado para publicación el 23 de noviembre de 2009.

\section{Correspondencia:}

Dra. Gladys Santos Falcón

Escuela Académico Profesional de Enfermería

Facultad de Medicina, UNMSM

Av. Grau 750. Lima 1, Perú

Correo-e: glads50@yahoo.com 